\title{
Participación y desarrollo local: Incidencia de las comunidades juveniles en el municipio de Sibaté-Cundinamarca, Colombia.
}

\author{
Edisson Gómez Pineda' \\ Departamento Administrativo Nacional de Estadística \\ Colombia
}

Fecha recepción: 29/05/20 I I

Fecha aceptación: |5/07/20II

\section{Resumen}

El concepto de participación acuñado con el paso de los años nos enfrenta a un sinnúmero de definiciones, aplicaciones y principios derivados de actores y fuentes teóricas disímiles. En concreto, el municipio de Sibaté, ubicado en el departamento de Cundinamarca, Colombia, se convierte en referente de estudio para explorar las dinámicas en las comunidades juveniles y su relación con el tema del desarrollo local. El ejercicio busca rastrear las incidencias de la participación comunitaria en el desarrollo local, para dilucidar panoramas y tendencias de acción por parte de la administración pública municipal y demás actores implicados, respecto a la planeación y ejecución de recursos públicos dirigidos a la población joven.

Palabras clave: Participación, desarrollo local, jóvenes, comunidades, municipio.

Participation and local development: The involvement of youth groups in the municipality of Sibate -Cundinamarca, Colombia

\begin{abstract}
Over the years the concept of participation has been given many different definitions, applications and principles originating from the perspective of different stakeholders and theoretical sources. The Municipality of Sibaté, located in the Department of Cundinamarca in Colombia, has become a case study for exploring the dynamics of youth groups and their relationship with local development issues. This article seeks to track the impact of community participation in its local development, in order to
\end{abstract}


ascertain an overview and future trends of policies implemented by the municipal government and other stakeholders with regard to the planning and execution of public expenditures targeted at youth.

Key words: Participation, local development, youth, community, municipality.

Participação e desenvolvimento local: a incidência das comunidades juvenis no município de Sibaté-Cundinamarca, Colômbia

\section{Resumo}

O conceito de participação cunhado com o passo dos anos faz nos enfrentarmos com diversas definições, aplicações e princípios derivados de atores e fontes teóricas diferentes. Em concreto, o município de Sibaté, localizado no departamento de Cundinamarca, Colômbia, se transforma em referente de estudo para explorar as dinâmicas nas comunidades juvenis e sua relação com o tema do desenvolvimento local. O exercício procura rastejar as incidências da participação comunitária no desenvolvimento local, para mostrar panoramas e tendências de ação por parte da administração pública municipal e de outros atores implicados, respeito ao planejamento e execução de recursos públicos dirigidos para a população jovem.

Palavras-chave: Participação, desenvolvimento local, jovens, comunidade, município. 


\section{Introducción}

En la actualidad, los estados democráticos del mundo tienden a interactuar más abiertamente con la sociedad, aspecto que se torna evidente en la vigencia que cobra la participación, toda vez que se hace más frecuente la expresión libre del pensamiento de los ciudadanos y de los medios de comunicación. En este contexto, resulta relevante reflexionar acerca del lugar que ocupa la participación juvenil, toda vez que a la juventud se le atribuye el futuro del desarrollo humano. Por tal razón, este ejercicio investigativo recurrió al análisis de la participación de comunidades juveniles del municipio ${ }^{2}$ de Sibaté, ubicado en el departamento de Cundinamarca, Colombia, y su intervención en el desarrollo local.

Para lograr lo planteado, nos inclinamos inicialmente por tratar de describir las dinámicas de la participación comunitaria de los habitantes de Sibaté, luego explorar algunos datos sobre el desarrollo humano en el municipio, desde el 2004 al 2009 , para finalmente dejar establecida una relación entre las dinámicas de participación comunitaria y algunos indicadores de desarrollo humano en el municipio de Sibaté.

Dada la importancia del actor social juvenil, debido a su rol en los procesos sociales de empoderamiento colectivo y de acción pública, se intentó dar una mirada a la constitución de comunidades juveniles y a su presencia en el escenario de lo público a la hora de tomar decisiones que afectan a toda la población del municipio. El procedimiento para rastrear el asunto consistió en una exploración y determinación teórica, posteriormente una de tipo documental y por último, una observación de la realidad a través del acompañamiento, la observación participante y la aplicación de un instrumento de recolección de información diseñado para el efecto, el cual permitió dilucidar las incidencias de la participación de los jóvenes.

\section{I.A propósito de la participación}

Aclarar y especificar términos como participación y desarrollo requirió de un ejercicio arduo de revisión bibliográfica, así como realizar una confrontación teórica que permitió abordar otros conceptos - como los de comunidad e incidencia.

Partamos con el macro concepto de participación

Inicialmente debe decirse que la conceptualización de la participación es polimorfa y sumamente prolífica, se diserta arduamente sobre una multiplicidad de sus dimensiones y acepciones, abarcando una vasta y divergente literatura sobre niveles y tipologías de participación, clasificadas éstas por actores, sectores, principios, etc. En el ámbito teórico, se destacan autores como Font, Budge, Gyford, Hopenhayn, Kendall, Held, Subirats y Bobbio. En el ámbito colombiano, figuran autores como Fabio

2 Nota del Editor: En este texto, la palabra “municipio" sirve para designar a una localidad o comuna y a sus habitantes, tal como lo utiliza la Federación Colombiana de Municipios.Ver <http://www.fcm.org.co/> 
Velásquez, Alejo Vargas, Jorge Luis Garay, Carlos Ariel Sánchez, Luis Alberto Restrepo, Luis Humberto Hernández, entre otros.

De otra parte, toda vez que el componente participativo es inherente al discurso político y está consignado en los distintos tipos de planes y programas de los gobiernos, la producción de textos de origen institucional, sólo en Colombia, es sumamente abundante. Los documentos que abordan el tema alcanzan un número indeterminado, en miles. No obstante, una concepción siempre debe verse desde una circunstancia, desde un punto espacial y temporal específico, atada a un contexto y asunto determinado.

De acuerdo con lo planteado por Rahnema (1996) al respecto de la participación, él establece una diferencia entre las formas transitivas de participación, las cuales se orientan a un objetivo o meta definidas; $y$ por contraste, las formas intransitivas, en las cuales el sujeto vive el proceso de participar sin perseguir un objetivo predefinido. Cuando uno está escuchando, amando, creando o viviendo plenamente su propia vida, uno toma parte sin buscar necesariamente el logro de un objetivo particular. Esta diferenciación nos permite plantear el papel principal de la forma transitiva de participación en el estudio, pues se trata de indagar y fortalecer esa participación activa, proyectiva y con trascendencia para los pueblos.

En este sentido, nuestro referente se relaciona más con la definición que da el Grup d'Aprofundiment Democràtic -GPD-, de la Mesa para los Derechos Sociales de Cataluña, quienes expresan que la participación es "La intervención consciente e intencionada de todo/a ciudadano/a en los asuntos públicos, a fin de instaurar una sociedad lo más libre y justa posible."(GPD, 2000, pp. 2-3). En dicha afirmación encontramos implícitos varios elementos que deben estar presentes en el ejercicio del participar, a saber: una conciencia y voluntad ciudadana; la intervención de todos, que implicaría una igualdad combinada con la libertad y la justicia que debe estar presentes en la sociedad. Según Aranguren

“Los mínimos en los que se asienta la cultura de la participación podríamos considerar que son los siguientes:

la cultura de la ciudadanía: nos sentimos miembros de una polis, de un territorio, de unas posibilidades que nos pertenecen, no como propietarios, sino como protagonistas de nuestra historia,

la cultura de la transformación: se participa en aquello que se desea transformar. Desde esta perspectiva, la participación representa un modo peculiar de profundizar en la democracia."(Aranguren, 2000, p. 02)

En este planteamiento encontramos otros elementos significativos, tales como una cultura participativa, que alude a las formas de ser, pensar, sentir y actuar que propenden por una participación transitiva que agrupa propósitos e individuos, donde la colectividad converge en un territorio, en una historia o en un ideal. Tenemos entonces la acepción de lo comunitario, la cual respecta a pequeñas colectividades donde sus integrantes se vinculan por poseer características o intereses en común. 
En el caso de los jóvenes, estas comunidades tienden a estar constituidas por la reunión de individuos que tienen subjetividades semejantes, donde los gustos de carácter cultural, las ideologías políticas, las tendencias sexuales, etc., son coincidentes entre varios sujetos. Estas comunidades son subgrupos del conjunto de los denominados “jóvenes", que para el caso son las personas que poseen una afinidad generacional y que tienen en común el territorio de hábitat del municipio de Sibaté. Óscar Rebollo, Coordinador y profesor del Postgrau Participació i Desenvolupament Sostenible de la Universidad Autónoma de Barcelona, plantea y trata de resolver la siguiente dicotomía:

"Participación de base individual vs. Participación de base asociativa. En los procesos sociales a los que nos referimos, los actores suelen ser colectivos, pues difícilmente podemos pensar en una persona "sola", que no actúe desde ninguna organización, ni con otros, ni para otros. La participación de base individual no existe. Lo que existen son participantes más o menos relacionados y organizados en la vida colectiva. O prácticas participativas que convocan a la gente mediante instrumentos que no son asociaciones." (Rebollo, 2003, p. 7)

En el postulado anterior encontramos elementos sumamente problemáticos, que atañen a las dinámicas participativas del caso específico. En el municipio de Sibaté, la dicotomía entre la participación de base individual vs. participación de base asociativa parece estar latente. Se estipula una presunta participación de base individual, llevada a cabo por sujetos, en singular, para la obtención de beneficios otorgados por actores institucionales. Por otra parte, la importante fuerza de la participación asociativa de las organizaciones del municipio se contrapone a este postulado. Más adelante trataremos de clarificar el escenario según lo consignado por las fuentes primarias utilizadas y lo observado en la realidad.

En la literatura explorada sobre participación, aparecen argumentos que por sí solos parecen ser paradójicos, pero que merecen especial atención por su carácter alternativo. Por ejemplo, Lo Vuolo, citado por Nora Britos ( 1999) señala:“... uno de los períodos de mayor perdurabilidad de las instituciones democráticas, se ve acompañado por el desmantelamiento de aquéllas políticas públicas cuya función tradicional era proveer de legitimación al poder político administrativo" (Britos, 1999, p. 4). Se podría inferir de esta afirmación que hay que abolir las acciones públicas que propendan a legitimar el poder político administrativo, para que así puedan perdurar las orientaciones democráticas. Este planteamiento es, incluso, lo que algunos actores juveniles del municipio de Sibaté avalan. Aunque esta proposición es muy relativa y polémica, nos conduce a indagar teóricamente por el papel de la participación en un escenario de este talante. Siguiendo nuevamente a Rahnema (1996), en relación con los tipos de participación, asumimos que hay "Formas manipuladas o teleguiadas de participación y formas espontáneas. En las primeras, los participantes no se sienten presionados para hacer 
algo, pero en realidad están guiados a realizar acciones que son inspiradas o dirigidas por centros fuera de su control." (Rahnema, 1996, p. 244)

Tendríamos así que las formas manipuladas, teleguiadas o espontáneas de participación podrían ser utilizadas de forma subalterna para legitimar las acciones de algunas instituciones. Esta dinámica de participación, muy problemática para la administración pública, nos lleva por ultimo a establecer unos referentes teóricos que nos permitan medir la participación en grados de profundidad o en niveles, de tal manera que se vea el cómo se participa.

Aunque existen múltiples formas de ordenar, categorizar y definir los niveles de participación, entre los que destacan por su idoneidad están los propuestos por la Corporación Autónoma Regional de Antioquia Corantioquia, quienes señalan los siguientes niveles: información, consulta, fiscalización, concertación, decisión y gestión. Sin embargo, se aclara que existen otros niveles de participación, por ende, éstos son susceptibles de ser reordenados y replanteados para cada caso en particular; no son una camisa de fuerza en los procedimientos ni son un dogma establecido, sino por el contrario, son planteamientos para enriquecer y reconstruir. Así, se toman como referentes para este estudio los niveles que plantea el Consejo Nacional de Planeación, por ser los más globales sectorialmente e idóneos para escudriñar los grados de profundidad e incidencia de la participación ciudadana en la institucionalidad pública colombiana.

En consecuencia, los referentes teóricos de niveles de participación ciudadana para este caso son:

- Participación concepto: “es el conocimiento, la idea o la comprensión o la razón.” (Forero, Cardona y Córdoba, 1999, p. 57) Respecto a las referencias normativas de la participación como idea o concepto, los autores citan la Constitución, en los artículos 2 y 78, donde se señalan las facilidades que se deben dar para la participación; también citan los artículos 133,40 y 259, de los cuales ellos transcriben "La comunidad impone mandato a los elegidos."(Ibídem, p. 5I) Este nivel de participación, a partir de la promulgación de la Constitución Política de 1991, tiene gran presencia, tanto en el discurso político como en los postulados de los documentos técnicos y administrativos de los distintos gobiernos regionales y locales. No obstante lo anterior, la superación de este nivel concepto hacia el siguiente nivel, denominado participación decisión, ha implicado multiplicidad de dificultades de carácter político, económico, cultural y organizacional, inherentes al anterior modelo de democracia representativa, que han impedido dar el gran paso hacia el nivel de participación decisión.

- Participación decisión: “una vez se piensa y se planea, se decide.” (Ibídem, p. 52) La participación decisión es ubicada por el Consejo Nacional de Planeación en segunda instancia, pero, dado que su estructura está conformada por tres verbos (pensar, planear y decidir), tiene un alto grado de complejidad; a nuestra 
consideración, para que pueda darse, ésta debe estar precedida por otro nivel de participación diferente al de concepto, cual sería el de participación gestión. Participación gestión: "se refiere a la participación de los ciudadanos en la elaboración de presupuestos y la ejecución de recursos.” (Ibídem, p. 53) Cuando un ciudadano ha participado coadyuvando a elaborar presupuestos y en procesos de planeación, se podría decir que ha adquirido un nivel de participación decisión, antes no. La participación gestión también hace referencia a un acompañamiento en la ejecución de los recursos, pero, para que este nivel de participación pueda darse, es indispensable la presencia de los elementos y principios fundamentales que requiere el fenómeno del participar, es decir que el ciudadano posea: información, igualdad, paciencia, libertad, comunicación, dinamismo, transparencia, respeto, responsabilidad y persistencia.

- Participación control: "sin los tres niveles anteriores, resultaría incompleta una disertación sobre control social en la gestión pública. Sin participación en la concepción, definición y ejecución de la gestión pública, su control desde la sociedad no tiene mucho impacto.” (Ibídem, p. 55) Ante este postulado, es de resaltar la afirmación de que no se puede hacer control de la gestión pública si el ciudadano no ha participado en todos los niveles anteriores de participación. Esto nos problematiza lo que se intenta hacer actualmente en Colombia, donde se pretende que el ciudadano sea un veedor cuando ha estado excluido de los demás procesos de concepción, gestión y decisión. Esta podría ser una de las principales causas por las cuales muchas veedurías ciudadanas funcionan mal o son inexistentes, pues no tienen los elementos necesarios para ejercer una participación control.

Finalmente, respecto de los niveles de participación, consideramos que los anteriormente planteados son los fundamentales. Es de considerar que otras acepciones, como los son, por ejemplo, el presunto nivel de participación informativa (rescatada en las rendiciones de cuentas de las instituciones públicas), no son integralmente niveles, pues estos hacen parte de los principios de la participación. Para el caso, el principio de la información no es un nivel de participación, pues ahí no existe una acción o efecto de participar; sólo hay un evento donde el ciudadano es un sujeto pasivo, casi exclusivamente receptor.

Otros niveles de participación, como la participación consultiva, no son viables de valorar como un nivel independiente de participación, sino tan sólo como uno de los requisitos indispensables para que se dé la participación en niveles como el de gestión y el de decisión. Este presunto nivel consultivo, no está constituido sólidamente con todos los principios del participar y tiende a no propender por un empoderamiento ciudadano, por una acción ciudadana con causa y efecto. Por el contrario, el ejercicio de la consulta ciudadana es un ejercicio propio de un sistema de democracia representativa utilizado tradicionalmente para la legitimación de acciones. Lo ideal es que la consulta sea un ejercicio fundamental que de paso a niveles de participación gestión y participación decisión. 
También se plantea la participación electoral como un nivel y además como tipología de participación. Esta participación tiene como característica hacer uso del ejercicio de la consulta y la elección de gobernantes para la representación. En las sociedades actuales es ampliamente utilizada como recurso de participación, para solventar problemas en poblaciones excesivamente numerosas que tienden a dificultar el ejercicio de la participación directa. La participación electoral ha sido el tradicional y principal instrumento de las democracias representativas del mundo para establecer los gobiernos y legitimar las estructuras estatales nacionales. No obstante su uso predominante, siendo un instrumento y una tipología de participación, no la consideramos un nivel pues indica una dimensión muy diferente. La participación de tipo electoral comúnmente es un noúmeno, el cual es:

“Utilizado en los esquemas democráticos contemporáneos, que se vale de la figura de la representación para la participación: El sujeto participa a través de un emisario que estará encargado de hacer llegar las inquietudes, planteamientos e intereses del representado a la esfera deliberativa o decisoria. El sujeto representado tiene un grado de participación, pues ha de tenerse como un alguien que hace parte cuantitativamente o cualitativamente de los representados, siendo inteligible como una abstracción existencial que participa en la deliberación o la decisión, donde puede ser objeto de inclusión o exclusión. No obstante la existencia de la participación como noúmeno, esta no es tangible, no es física, no es una acción u efecto del sujeto como tal dentro de la esfera participativa.” (Gómez, 2009, p. 2)

Una vez especificada la existencia y el carácter del noúmeno participativo que es inherente a la participación electoral para la elección, fácilmente podemos inferir que tampoco es posible incluirlo en los niveles de participación estándar, pues estos son niveles con una dimensión fenoménica de participación, lo cual los hace claramente diferenciales. Sin embargo, al ser la participación electoral también un instrumento, tenemos la discrecionalidad de efectuar modificaciones y mutaciones a ese instrumento en todos los escenarios, pues con su actual utilización masiva para la elección de gobernantes, se puede reorientar con la intención de que sea un instrumento que facilite todos los niveles de concepción, gestión, decisión y control participativo de una forma más real.

Con lo planteado hasta el momento, contamos con unos referentes teóricos sobre la participación que nos permitieron identificar y comprender los enunciados para el caso. Supimos a qué se hace alusión cuando se expresa, por ejemplo, una participación transitiva, de nivel gestión, de forma manipulada o de carácter noúmeno con base asociativa, etc. Lo anterior sirva como precedente para desarrollar el primer objetivo especifico, que trata de la descripción de las dinámicas de participación de las comunidades juveniles de Sibaté. 
Una vez efectuada esa descripción, se procederá a teorizar y caracterizar el desarrollo del municipio y a establecer las relaciones existentes entre la participación y el desarrollo local.

\section{Descripción de las dinámicas participativas juveniles en sibaté}

Para efectuar la descripción de las dinámicas participativas juveniles en Sibaté, se utilizaron básicamente dos instrumentos: la encuesta y las fichas de observación.

A continuación, se hace relación de los resultados obtenidos en la encuesta aplicada a los jóvenes participantes en acontecimientos musicales en el marco del Mes de la cultura Sibatense, en el año 2009, los cuales fueron una serie de eventos organizados por la administración pública municipal.

Los encuestados fueron personas que tuviesen entre 14 y 26 años, a quienes la ley colombiana No 375 de 1997 les reconoce la condición de juventud. Se tomó como universo la población total de 6.884 jóvenes que habitaban el municipio para el año 2009 según estadísticas oficiales. Se realizó un muestreo aleatorio sistemático en los eventos, alcanzando por oportunidad una proporción del 4,45\% respecto de la población total, manteniendo una muestra con distribución por genero simétrica y con cobertura global de 306 participantes que reunían legalmente la condición de jóvenes. Los resultados finales de la encuesta arrojaron un margen de error de 6, I\% y un nivel de confianza de $97 \%$.

El cuestionario fue aplicado en físico a jóvenes participantes en los eventos, quienes de forma voluntaria, libremente y por escrito proporcionaron las siguientes respuestas:

I. ¿Entre cuál de los siguientes rangos de edad se encuentra usted? Con esta pregunta se pudo observar que la mitad de los asistentes tenían más de 22 años de edad, seguido proporcionalmente por jóvenes entre 14 y 17 años. Esto podría mostrarnos una tendencia evolutiva del interés de los jóvenes por participar en estos eventos, mas concentrada al principio de su juventud y también al finalizar esta etapa, luego de cierto tipo de aislamiento entre las edades de 18 a 21 años.

2. Nivel de educación. Casi la mitad de los asistentes (44\%) son bachilleres. Esto nos podría indicar que los jóvenes que egresan de la secundaria ven la necesidad de verse incluidos en proyectos y programas de carácter cultural, en los cuales puedan interactuar con sus amigos y personas de su misma generación. Seguidamente, encontramos en menor proporción a los estudiantes universitarios (31\%) y luego, a los estudiantes de secundaria, quienes aprovechan estos espacios como complemento a sus actividades académicas curriculares.

3. ¿Cuál es el estrato socio económico identificado en los servicios públicos de su vivienda? Observamos que la gran mayoría de los jóvenes participantes (81\%) pertenecen a estrato dos y el resto (19\%), al estrato tres. El estrato 
socioeconómico, utilizado como sistema de identificación del entorno de vivienda, actúa más como un indicador urbanístico que como un indicador de desarrollo propiamente dicho; un entorno de hábitat de grado medio - bajo, es representativo de las pocas comodidades existentes en las viviendas. Esto también nos indica que mayormente son los estratos bajos los que asisten a estos eventos, los cuales no tendrían ningún tipo de impacto en poblaciones de estratos altos.

4. ¿En qué zona del municipio vive? Encontramos en esta respuesta aspectos sumamente problemáticos: más de la mitad $(60 \%)$ de los asistentes a estos eventos son personas foráneas al municipio. Esto nos indicaría que las actividades que realiza la administración pública municipal no impactan en la población local, sino que benefician a personas de otros lugares diferentes al municipio. La cercanía con Bogotá facilita que se filtren fácilmente los actores más interesados en el tema, quedando por fuera los jóvenes del municipio. Otro asunto que llama la atención, es la total ausencia de comunidades juveniles rurales en estos eventos. Esto nos indicaría que los jóvenes del campo están completamente excluidos de los programas y proyectos de carácter colectivo implementados por la alcaldía.

5. ¿ iHa pertenecido en algún momento a una organización social o cultural? Se evidencia también la existencia de un grado de asociatividad muy representativo (3I\%). No obstante, la mayoría sigue estando sin pertenecer a ninguna organización en concreto (69\%).

6. ¿De qué tipo era la organización a la que pertenecía? Respecto del $69 \%$ de los que se han organizado alguna vez, el $60 \%$ considera sus organizaciones de carácter público, ya sea por sus acciones, sus intereses o lineamientos jurídicos. Esto evidenciaría una tendencia de interés juvenil por lo público.

Dado que indagamos por las incidencias de la participación juvenil en el desarrollo local, se intenta especificar el concepto “incidencia”. Es importante partir enunciando que esta es una acepción no muy elaborada en el ámbito académico concerniente a ciencias sociales y administrativas, siendo más propio de las ciencias medicas. Esta situación ocurre principalmente debido a que en el ámbito de las ciencias sociales y administrativas, se tiende a reemplazar la palabra por términos parecidos - no sinónimos-, sin valorar a profundidad su relevancia.

Para el presente caso, se considera que utilizar la palabra "incidencia" es apropiado en cuanto esta es un referente conceptual específico, inherente a las dinámicas del ejercicio participativo en los regímenes democráticos. En específico, cabe traer a colación la definición que otorga el instituto Internacional de Medio Ambiente y Desarrollo - América Latina -IIED-, sobre la incidencia: 
Es un proceso que contempla la realización de un conjunto de acciones políticas de la ciudadanía organizada, dirigidas a influir en aquellos que toman decisiones y lograr cambios en las políticas públicas. (IIED-AL, 2006, p. I2)

Si observamos el termino en el contexto contemporáneo de los Estados Latinoamericanos, se puede afirmar que la incidencia o no incidencia de unos actores sociales determinados en la toma de decisiones públicas, es un factor preponderante a la hora de sopesar la verídica existencia de un sistema democrático pluralista, que da cabida al reconocimiento e interacción de los distintos agentes sociales en relación con los entes gubernamentales.

En este orden de ideas, alguien incide cuando a través de sus acciones o ideas influye en la toma de decisiones públicas. No obstante esto, consideramos que la incidencia también puede verse reflejada en los impactos de la decisión publica, e incluso en la misma situación social por la cual se interactúo. De esta manera,y de forma subjetiva, podríamos plantear el concepto de incidencia, asumida ésta como el efecto, acontecimiento, acción, asunto o proceso que sobreviene a una eventualidad o fenómeno, ya sea como consecuencias contempladas o externalidades imprevistas, producto de las influencias que los agentes han tenido en los procesos de interacción publica, ya con los representantes del gobierno local o con los demás agentes sociales implicados en la planeación, gestión y decisión de políticas públicas para el desarrollo local.

El indagar por la incidencia de la participación en el desarrollo local, nos lleva a preguntarnos también por una acepción del desarrollo.

\section{¿Qué se entiende por desarrollo?}

Sobre el desarrollo local realizamos una breve indagación de una manera heurística, donde el pensamiento desplegado por los autores fue consultado documentalmente, pero solo acogido y referenciado de forma subjetiva de acuerdo con lo pertinente y relacionado a nuestro contexto en específico; pues, inevitablemente, como diría Dudley Seers, citado por Boisier, “..."desarrollo” es un concepto normativo, lleno de juicios de valor.” (Boisier, 2002, p. 4) En este sentido, se referencia taxativamente sólo algunas de sus múltiples acepciones, las que se traen a colación con el ánimo de observar desde esas perspectivas las dinámicas del municipio de Sibaté.

Como la mayoría de los municipios colombianos, en Sibaté la extensión de área rural es muy superior a la urbana; no obstante, la tendencia mundial de hábitat que propende por la vida en las ciudades hace que la mayoría de la población se concentre en las cabeceras urbanas. Estas tendencias están por si mismas ligadas a un modelo de desarrollo. Siguiendo a Kay, que analiza seis macro enfoques de desarrollo: "Estructuralismo, modernización, dependencia, neoliberalismo, neo estructuralismo y estrategias de vida..." (Kay, 2007, p. 50), nos planteamos que enfoques como el de estrategias de vida es el que más propende por estudios de carácter sociológico y antropológico; es decir, es un enfoque que analiza prácticas, comportamientos, di- 
námicas sociales, etc. mas relacionadas con una noción directa del desarrollo, de las necesidades y menos con temas económicos o financieros. Por ende, la restricción de acceso a servicios públicos básicos, la falta de insumos mínimos y la falta de capacidades adquisitivas que impiden llevar dignamente una existencia, se constituirían en problemas de pobreza o de falta de desarrollo.

Sen define el subdesarrollo como "...la privación de capacidades básicas y no meramente como la falta de ingresos, que es el criterio habitual que identifica la pobreza" (Sen, 2000, p. I I4). De acuerdo a este postulado, tenemos que la medida de pobreza basada en el nivel de ingresos no es idónea, puesto que, por ejemplo, dos dólares en determinado contexto pueden ser inútiles cuando no hay servicios que comprar en entornos excluidos del mercado donde la moneda no funciona, o en contextos en los cuales los bienes son muy costosos y los dos dólares resultan totalmente insuficientes para acabar con el hambre, la ignorancia y la enfermedad de las personas.

Celso Furtado, citado por Boisier dice que "El verdadero desarrollo es principalmente un proceso de activación y canalización de fuerzas sociales, de avance en la capacidad asociativa, de ejercicio de la iniciativa y de la inventiva. Por lo tanto, se trata de un proceso social y cultural, y solo secundariamente económico." (Boisier, 2003, p. I) Con esta afirmación, vemos que las poblaciones, las comunidades y las personas, en general, han de ser las protagonistas de su propio de desarrollo, pues la iniciativa, la inventiva y la capacidad asociativa en los distintos actores de la sociedad son elementos fundamentales para la evolución hacia lo que este autor denomina un "verdadero desarrollo". Sin embargo, es de considerar que contemporáneamente autores como Sckopol (1995), sostienen que el Estado debe regresar al primer plano, tanto en los estudios académicos como en las dinámicas sociales que demandan su presencia. Otros autores, de líneas de estudio como el institucionalismo, van por esta misma línea argumentativa. Entonces, resulta evidente que es necesaria la presencia de un Estado que gestione políticas, procesos y procedimientos tendientes a mejorar el nivel de vida de las personas.

Al respecto, Corragio plantea que es el paradigma del desarrollo local el que propone otras escalas y otros actores del desarrollo: "un gobierno local democrático-participativo aparece en algunas propuestas como una condición indispensable adicional a la presencia de una densa red de actores de la sociedad civil." (Corragio, 2004, p. 80) El desarrollo parece ser, así, uno de esos elementos teleológicos, de causa final, por la cual existen los Estados. Bajo este precepto, el accionar de las administraciones públicas debería estar orientado a obtener mejores condiciones de vida.

¿Qué datos nos dicen algo sobre el desarrollo local de los jóvenes de Sibaté?

Ya hemos establecido una relación teórica inexpugnable, consistente en que la participación es un elemento clave para mejorar los niveles de desarrollo. La pregunta en específico, indaga por la incidencia de las participaciones juveniles en el desarrollo. El intento por caracterizar una situación social está supeditado a lo que nos muestran las apariencias y la veracidad de la información contenida en los documentos oficiales 
consultados. Ciertamente las apariencias pueden resultar engañosas, dada una tendencia subalterna que informalmente existe, la de proferir en el papel cosas distintas a la realidad. Este obstáculo ha tratado de ser superado con las visitas de campo para la verificación de la realidad, que será informada con posterioridad. Para esta sección nos basamos en las fuentes y el análisis de la institucionalidad pública estatal que trata asuntos en este territorio.

A continuación, básicamente se trata de hacer la descripción situacional de los principales componentes del desarrollo, en especial del acceso de las comunidades a bienes y servicios básicos. La abordamos explorando los principales componentes de salud, educación, agua potable y saneamiento básico, vivienda etc., es decir, de acuerdo a la división sectorial que se da desde la administración pública.

Iniciamos enunciando los principales datos del sector salud: de acuerdo a las proyecciones poblacionales del Departamento Administrativo Nacional de Estadística (DANE), Sibaté debe tener en el año 2009 un total de 34.330 habitantes, de los cuales I7.I39 (50.01\%) son hombres y I7.19I (50.07\%) mujeres. Lo cual significa que las proporciones de género son casi simétricas. Entre 2005 y 2009, la población de Sibaté creció en 2.655 personas, a una tasa del $2.09 \%$ anual.

Los jóvenes representaban un $24.76 \%$ (6.884) de la población total, con una distribución por genero similar.

\section{Gráfico I. Utilización de los servicios de salud de Sibaté, por edad}

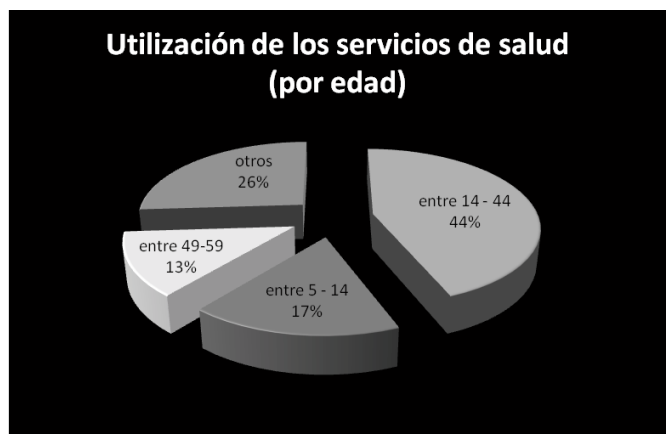

Fuente: Secretaría de Salud de Sibaté, 2007

Con respecto a la utilización de los servicios de salud, se registra un un total de 3 I.I85 consultas, las que contemplan: medicina general, especializada, enfermería, nutrición, psicología y optometría, en las diferentes instituciones prestadoras de salud (IPS) ubicadas en el municipio. De la mencionada cantidad, I 3.830 son consultas realizadas por personas entre 14 y 44 años. Es decir, que un $44 \%$ del total de consultas las realiza población entre en este rango margen de edad. Lo cual significaría que entre jóvenes y personas que no han entrado en la vejez son quienes más demandan los servicios de salud, comparativamente con los porcentajes de otros grupos erarios, 
que son menores. Enseguida siguen los niños de 5 al 4 años, con un 17\%, los adultos de 49 a 59 años, el 13\%, etc. Es importante considerar que al existir una demanda social, es necesaria una oferta suficiente preferiblemente con criterios de gratuidad.

Otra cifra importante en el sector salud concerniente a la población joven, corresponde a los indicadores de maternidad y embarazo que presenta la Secretaria de Salud de Sibaté, para el año 2007.

\section{Gráfico 2. Embarazo en adolescentes, en Sibaté}

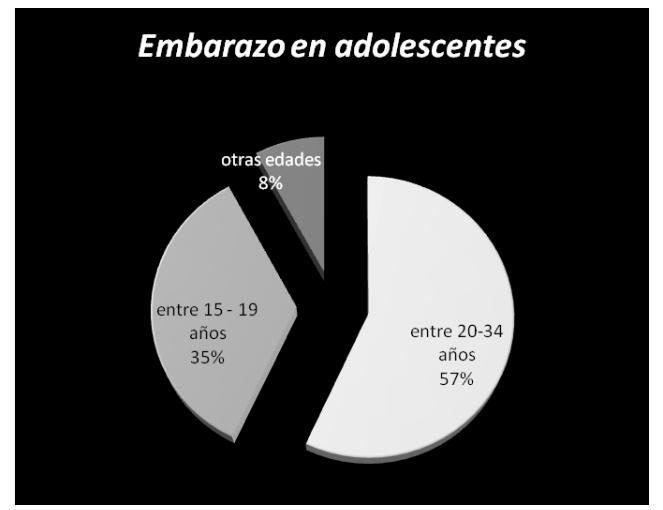

Fuente: Secretaría de Salud - Sibaté, 2007

El informe es anotado en el plan de desarrollo vigente y dice textualmente:

“El grupo de 20 a 34 años representa el $57 \%$ de las gestantes, I 26 están en el rango de los 15 a 19 años, equivalente al 35\%, lo que indica un alto porcentaje de embarazo en adolescentes. Esto plantea la necesidad de fortalecer los programas de prevención..." (Alcaldía de Sibaté, 2007, p. I5)

Las referencias anteriores nos permitieron detectar puntos críticos que, por su carácter álgido, muestran la necesidad de atención especial por parte de la administración pública, para amortiguar las respectivas problemáticas con las estrategias más adecuadas para mejorar las condiciones de salud pública y prevenir eventualidades no deseadas y poco proclives para la potenciación de la participación ciudadana y el desarrollo local.

El planteamiento de nuevas posibilidades o estrategias de superación de dificultades no podrá excluir, de ninguna manera, el componente participativo. Más adelante indagaremos por el mismo, en los procesos adelantados hasta el momento.

En la información contenida en las siguientes tablas tenemos la distribución de la cantidad de población que recibe servicios de forma prioritaria por parte del municipio, dividida de acuerdo al lugar habitacional. 


\section{Tablas I - 2 - 3 - Población beneficiaria de servicios públicos en el} municipio de Sibaté, 2009

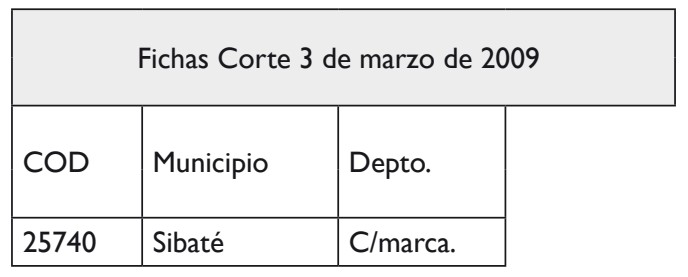

\begin{tabular}{|l|l|l|l|l|}
\hline \multicolumn{5}{|c|}{ Urbano } \\
\hline 1 & 2 & 3 & 4 y mas & Total \\
\hline 588 & 3.050 & 1.114 & 5 & 4.757 \\
\hline
\end{tabular}

\begin{tabular}{|l|l|l|l|l|}
\hline \multicolumn{5}{|c|}{ Centro Poblado } \\
\hline$I$ & 2 & 3 & 4 y mas & Total \\
\hline 157 & 533 & 38 & 5 & 733 \\
\hline
\end{tabular}

\begin{tabular}{|l|l|l|l|l|}
\hline \multicolumn{5}{|c|}{ Rural Disperso } \\
\hline 1 & 2 & 3 & 4 y mas & Total \\
\hline 302 & 711 & 121 & 24 & 1.158 \\
\hline
\end{tabular}

\begin{tabular}{|l|l|l|l|l|}
\hline \multicolumn{5}{|c|}{ Total } \\
\hline $\mathrm{I}$ & 2 & 3 & 4 y mas & Totales \\
\hline 1.047 & 4.294 & 1.273 & 34 & 6.648 \\
\hline
\end{tabular}

Fuente: Sisbén, 2009

Si analizamos las tablas de forma horizontal, es decir comparándolas entre sí, tenemos que 4.757 personas, equivalente al 71.56\% de los beneficiados con este servicio, habitan en zona urbana. El 17.42\% habita en zona rural y el II.02\%, en otros centros poblados. Comparativamente con las cifras de atención y servicio antes mencionadas, esto nos ratificaría que, en efecto, la condición de habitación urbana favorece el acceso y la participación en los servicios de salud.

Si realizamos un análisis vertical de la tabla de los totales, correspondientes al 2009 , tendríamos que el $64.59 \%$ de la población del municipio con vinculación priorizada en el Sistema de identificación de potenciales beneficiarios de programas sociales (Sisbén), está clasificada en nivel dos, lo cual, según las fichas técnicas del Departamento Nacional de Planeación (DNP) para la administración del Sisbén, las personas con nivel dos tendrían derechos importantes como un régimen subsidiado en salud, en la red hospitalaria para atención, entre otros. 
Respecto a temas de educación, según el último censo de educación (2005), tenemos que el $73.9 \%$ de los aproximados 7.000 jóvenes que viven permanentemente en Sibaté, cuenta con estudios de enseñanza secundaria y el $15.4 \%$ ha ingresado a la educación superior, pero, sólo un 7.5\% ha culminado dicho proceso.

La situación en relación al sector vivienda, es presentada por la institucionalidad pública así:

"Encontramos que I.21 8 familias vive en condiciones de hacinamiento (6 personas o más por vivienda) que corresponden al $17.1 \%$ de los 7.126 hogares presentes en el municipio.

Así mismo, se puede notar el alto número de hogares que pagan arriendo, 3.354 familias que corresponden al $47.06 \%$, concentrándose en el casco urbano en su mayoría con 2.637 familias equivalentes al $37 \%$. Por otra parte, las 4.546 viviendas de la zona urbana albergan 5.201 hogares; en el sector rural Las 1787 viviendas albergan 1925 hogares. Mostrando un déficit de vivienda del $12.59 \%$ y $7.17 \%$ respectivamente, sumando un total de 793 hogares."(Alcaldía de Sibaté, 2007, p. 3 I)

Para nosotros, el hacinamiento y la carencia de un techo propio no son buenas condiciones de vida e indican bajos niveles de desarrollo. Si sumamos el porcentaje de familias en hacinamiento con las que pagan arriendo, tendríamos entonces que el 64. $16 \%$ del total de familias no están en las mejores condiciones. Detectamos, entonces, serios problemas de vivienda especialmente en la zona urbana.

“Adicionalmente, encontramos déficit en los servicios sanitarios de las viviendas, I 10 viviendas carecen de este servicio principalmente ubicadas en la parte rural." (Ibídem, p. 32 )

El déficit de servicios sanitarios en zona rural no sólo significa déficit en la vivienda, sino un déficit en el saneamiento básico y en el agua potable.Vemos que un sector está vinculado al otro, con problemas comunes que hay que solucionar. De acuerdo a la categorización del Sisbén, tenemos que los niveles uno y dos tendrían derecho a subsidios de vivienda de interés social para adquisición, construcción o mejoramiento.

Por su parte, la alcaldía municipal, en información proporcionada por la oficina de control interno, respecto de los indicadores por dependencia al respecto muestra los siguientes datos:

\section{Tablas 4 - 5}

\begin{tabular}{|l|l|l|}
\hline 5.I. Indicador & 5.2. Resultado (\%) & 5.3.Análisis del resultados \\
\hline
\end{tabular}




\begin{tabular}{|c|c|c|c|}
\hline $\begin{array}{l}\text { LA VIVIENDA QUE MERECEMOS } \\
\text { I-DIGNIFICANDO NUESTROS } \\
\text { HOGARES } \\
\text { Mejorar la calidad de las viviendas } \\
\text { en un } 50 \% \text { de las familias que lo } \\
\text { requieren. (42\%) } \\
\text { 2-NUEVO HOGAR NUEVA VIDA } \\
\text { Reducir el déficit de vivienda } \\
\text { urbana en un I6\% y rural en un } \\
58 \% \text { (I } 00 \%\end{array}$ & $\begin{array}{l}\text { I-Realizar } 280 \text { mejoramientos } \\
\text { de vivienda en niveles I y II del } \\
\text { Sisbén. Realizar I } 20 \text { beneficios } \\
\text { de vivienda saludable. Realizar } \\
80 \text { beneficios de techo digno. } \\
\text { 2-Formulación e inscripción del } \\
\text { proyectos en el BMPPIN }\end{array}$ & $71 \%$ & $\begin{array}{l}\text { I-En el primer } \\
\text { proyecto, la } \\
\text { convocatoria } \\
\text { para la compra } \\
\text { de materiales se } \\
\text { declaró desierta. }\end{array}$ \\
\hline
\end{tabular}

Fuente:Alcaldía de Sibaté, Plan de desarrollo municipal 2008 - 201 I.

Al respecto de la vivienda, parece que se ha hecho un poco más de énfasis y prestado más atención desde la administración pública. Pero, respecto a las comunidades juveniles en específico, no se referencian indicadores ni ningún tipo de datos de indigencia, necesidades ni nada por el estilo.

Dado nuestro interés por la participación juvenil frente a esos indicadores, estas dudas tal vez se disipen más adelante en la observación e indagación de campo realizada. Por ahora podemos inferir dos cosas: los sectores están entrelazados entre sí: vivienda con saneamiento básico y este a su vez con infraestructura; dato que observamos en la destinación presupuestal referenciada así: "Infraestructura en saneamiento básico y agua potable: \$404.000.000” la cual se supone será un complemento para mejorar las condiciones sanitarias y los equipamientos para brindar servicios de salud y educación. (Fuentes: DANE, 2005; SISBEN, 2007; y Plan de desarrollo municipal, 2008 - 20II.)

Hay que aclarar que fuentes como el Sistema de identificación de beneficiarios (Sisbén), son sólo bases de información de carácter exclusivamente nacional, que no son exactamente unos indicadores de desarrollo, pero son datos que si permiten dilucidar un panorama general respecto al acceso a estos servicios y sirven para orientar las acciones de la administración pública en diferentes sectores del desarrollo de estas comunidades.

Es importante mencionar que existe un problema en la recopilación de datos, pues existen múltiples informes de distintas entidades públicas que presentan datos diferentes sobre un mismo asunto, en un mismo periodo. (Ejemplo: El Sisbén dice que en 2005 el $76 \%$ de los jóvenes habían terminado secundaria, mientras el DANE dice que era el 72\%). Diagnosticar rigurosamente las comunidades juveniles resulta sumamente difícil, dado que las clasificaciones del Sisbén y de otras bases de información que se basan en unidades trazadas por hogares o por cabezas de hogar, es decir, por padres o responsables económicos, se traduce en que los jóvenes comúnmente no son clasificados o referenciados de forma independiente en algunos de los sectores e indicadores de desarrollo humano. Además, es difícil observarlos, dada la movilidad inherente a la condición joven, que oscila repentina 
y esporádicamente entre estadios como la dependencia-independencia económica, la educación continuada-descontinuada, la constitución-disolución de nuevos hogares o unidades familiares. Es complicado referir con exactitud el acceso de forma específica de los jóvenes a la vivienda y las condiciones de sanidad, etc. Aunque se detectan intentos por acoplar los diagnósticos a estándares de desarrollo mundiales, como los objetivos de Desarrollo del Milenio, datos concretos bajo estos esquemas no fueron encontrados.

Se efectuó una exploración de los servicios ofrecidos por la Alcaldía municipal de Sibaté, dirigidos a la juventud. A través de un ejercicio de indagación personal, telefónica y electrónica por cada una de las dependencias de dicha institución, se encontró un alto grado de evasión por parte de la administración, donde se postergaron las respuestas, se excusaron por inexistencias y se aludió a problemas técnicos.

Sin embargo, resalta el inventario de servicios dirigidos a las comunidades de jóvenes que plantea la Secretaría de desarrollo social de la Alcaldía. Los servicios relacionados que ofrece son:

"I. Equipo de apoyo psicosocial para las instituciones educativas (IED),

2. Transporte para los niños que viven a más de tres Kilómetros de su institución educativa,

3. Alimentación escolar para el 100\% de primaria, de las instituciones oficiales del municipio,

4. Escuelas de formación Artística, Cultural y de Artes afines,

5. Co-financiación para cupos de hogar infantil (Pitufos, los amiguitos y madres comunitarias),

6. Formación a población en desplazamiento, en convenio con el SENA,

7. Coordinación del programa de alimentos dirigida a población en condición de desplazamiento." (Alcaldía de Sibaté, 2007, p. 86)

Existen otros programas y proyectos que de forma dispersa se encuentran plasmados, pero, de los cuales no se obtuvieron resultados de avances. Adicionalmente, la administración municipal presenta un inventario de servicios a jóvenes que no son directamente prestados por la entidad: incluyen programas como el crédito para la educación, que es prestado por el Instituto Colombiano de Créditos y Estudios Técnicos en el Exterior (Icetex); mencionan otro programa de asistencia y apoyos que formalmente son prestados por otras entidades de orden superior, como son los ministerios. Estos servicios, en muchos casos, no son prestados por poca presencia institucional foránea o por poca participación de los jóvenes en estos procesos, a causa de un desconocimiento de los mismos o poca viabilidad en la aplicación. 


\section{III. ¿Qué incidencias o relaciones existen entre la participación de los jóvenes y el desarrollo local en sibaté?}

Durante el transcurso del segundo semestre de 2009, se visitó semanalmente el municipio de Sibaté, tratando de asistir a todas las reuniones de las organizaciones en que participan los jóvenes.

Para efectuar un seguimiento de las dinámicas participativas, su relación con la administración pública y su incidencia en el desarrollo, se utilizó como instrumento de recopilación y registro de información en las respectivas salidas de campo, una ficha de observación de eventos diligenciada por cada investigador. Esta ficha se diseñó tratando de plasmar descripciones de las categorías claves de estudio en los distintos escenarios: se describía la participación, los actores y las dinámicas de acción. Aunque se trató de seguir una observación rigurosa y neutral, la complejidad de los escenarios reales es multidimensional. Es decir, las conceptualizaciones, observaciones e inferencias realizadas en una salida de campo, teorizadas y sistematizadas racionalmente, están limitadas por un grado de percepción y razonamiento subjetivo o intersubjetivo, donde no resulta posible dar cuenta de toda la complejidad de la realidad. La multiplicidad de percepciones es directamente proporcional a la multiplicidad de dimensiones del ser; es decir, la configuración de una realidad sólo puede ser abarcada en su totalidad cuando se observan la totalidad de los puntos de vista desde donde es posible concebirla. No obstante lo anterior, la observación y sistematización de información a través del mencionado instrumento, fue el elemento clave que permitió hacer seguimiento a los hechos y dio luces sobre lo realmente ocurrido.

En la observación realizada, se abordaron escenarios de carácter típico, tratando de eludir la excepcionalidad, con el ánimo de hacer un análisis de la etnografía cotidiana en las prácticas juveniles, en el cual la predisposición del comportamiento rastreado estuviese suprimida. De esta manera se podría realizar un seguimiento y una observación en la cual la actitud de teatralidad condicionada no modifique la espontaneidad de los actores ni el panorama de la escenificación de realidad.

En las salidas a terreno efectuadas, resultó evidente una activación de los actores en el municipio, un ejercicio participativo de base asociativa, inicialmente de forma espontánea, en escenarios deliberativos y de concertación grupal. La existencia de una Casa de la juventud en el municipio, se mostró como un recurso físico muy favorable para convocar y reunir a los jóvenes. En este sentido, se observó una agrupación de actores relativamente constante -casi todos los fines de semana, exceptuando los vacacionales y otros ocasionales-, donde convergían al diálogo jóvenes representantes de distintas organizaciones culturales y ambientales juveniles. Se observó nula presencia de jóvenes independientes, que no fuesen integrantes de alguna organización. Aunque en los temas de discusión se planteaba la inclusión de actores -como los jóvenes de hábitat rural y la inclusión de cualquiera- en la práctica se vio ratificado el postulado del teórico Rebollo (2003) ya enunciado, quien argumenta la imposibilidad de una participación de base individual. En estas eventualidades y comúnmente con un representante de la alcaldía, se observó un escenario participativo 
en apariencia ideal, donde los esfuerzos por implementar niveles de participación concepto, gestión, decisión, control, etc. fueron muy valiosos.

Se observó, además, que existe otra participación de nivel gestión, que parece ser de base individual, pero que está viciada por pugnas entre los sujetos por obtener acceso a beneficios y recursos públicos. Este fenómeno también se presenta con la participación de base asociativa: las distintas agrupaciones juveniles se confrontan entre sí, con el propósito de obtener mayores recursos y ventajas para realizar sus propios eventos, en los cuales sólo participan las personas vinculadas a la organización. En sus palabras siempre se manifiestan el respeto por el otro y el apoyo a las organizaciones y sujetos que son y piensan diferente, pero, en los eventos, esto no es muy visible.

De acuerdo a lo anterior, vemos que la participación si ha contribuido a fortalecer un desarrollo acorde con la definición ya citada de Celso Furtado, la cual resalta los avances en la capacidad asociativa, la inventiva y la canalización de fuerzas en el fortalecimiento de la activación cultural y social. No obstante, esta misma participación ha tenido una incidencia muy limitada respecto a un desarrollo con noción más técnica, es decir respecto a indicadores, cifras y problemáticas de carácter económico.

Aunque existen principios de participación presentes, como la información en algunos jóvenes, e incluso manifestaciones verbales respecto de algunos problemas como la situación ambiental, la pobreza, la desigualdad y la maternidad en jóvenes, estos conceptos tienden a no ser incidentes en el ámbito decisorio de la administración pública. La planeación y el manejo presupuestario en temas juveniles no culturales, sigue siendo de manejo tecnocrático por parte de la administración pública.

\section{Conclusiones}

En el estudio efectuado se pudo detectar algunos problemas que respectan a la amplitud de la cobertura de servicios y atenciones brindadas por parte de la Alcaldía de Sibaté a la población joven del municipio. Los datos sobre desarrollo local nos mostraron desigualdades en los accesos a servicios, beneficios y procesos participativos de las comunidades juveniles, especialmente condicionados por el entorno de hábitat: quienes habitan en zonas rurales tienden a disminuir, por estar más aislados, su participación en los procesos de deliberación, concertación y decisión que se llevan a cabo.

En lo que respecta a niveles de participación, se encontró principalmente el nivel de gestión, nivel que es ejercido con una base asociativa de participación por parte las organizaciones existentes en el municipio, las cuales están constituidas principalmente por comunidades agrupadas por afinidades de carácter cultural, donde los sujetos se asocian principalmente para compartir gustos musicales y demás ideales de existencia. El nivel gestión se presenta entre las comunidades juveniles y la alcaldía municipal, principalmente de forma ascendente, desde abajo hacia arriba, donde los sujetos asociados a comunidades juveniles específicas interactúan con el gobierno lo- 
cal en la gestión de presupuestos para la implementación de proyectos culturales que favorezcan sus intereses en particular, grupales o comunitarios. Aunque parece ser una acción social con trascendencia, el problema de estos procesos es la exclusión de otras comunidades juveniles y, especialmente, la exclusión de sujetos no asociados a organizaciones determinadas, para quienes el mismo proceso se convierte en una participación nivel concepto, que no es de ninguna manera incidente.

Si bien es cierto que han existido esfuerzos por incluir a todas las comunidades y sujetos, tanto individuales como colectivos, tratando de fomentar un mutuo reconocimiento de la diferencia entre sí, estos procesos son tildados por algunos actores como formas manipuladas o teleguiadas de participación. Ello significa que el proceso se da de forma descendente, desde arriba hacia abajo, de tal manera que el gobierno ejecuta e implementa estas actividades sólo con actores específicos; esto con el ánimo de menguar las presiones sociales y presentar resultados de una gestión democrática y participativa, la que acontece sólo en el ámbito cultural.

Dada la evidente des-favorabilidad de los jóvenes que habitan en la ruralidad, quienes tienen menor acceso y calidad en los servicios básicos de educación, salud, agua potable, infraestructura, transporte y dado que también están en condición de desventaja por tener un grado inferior de asociación y mayor aislamiento en términos espaciales, se hace visible que la pluralidad democrática no es totalmente incluyente. Hay públicos subalternos que se encuentran en segundo plano, en condiciones de desigualdad.

Finalmente, desde la perspectiva de la institucionalidad pública, se observa un modelo de política y de gasto público focalizado, que difícilmente tiene impacto en toda la población. En la política pública de juventud y en el plan decenal de juventud se establecen unos postulados de inclusión y desarrollo hasta el 2016, que desde la retórica sería un ideal materializar. Se ve la necesidad de diseñar un programa que universalice el acceso de los jóvenes a los escenarios participativos para que empiecen a incidir, que evite la apropiación de sólo algunas organizaciones particulares de los espacios disponibles, que permita hacer inversiones que tengan impactos en toda la población de Sibaté. De esta manera se podría evitar que actores foráneos sean los beneficiarios, dejando así excluidos a los natales y habitantes del municipio, como ha ocurrido en algunos eventos y proyectos de tipo cultural y recreativo.

\section{Bibliografía}

Alcaldía de Sibaté (2007, 2008a) Documentos de diagnostico por Secretarias del plan de desarrollo municipal 2008 - 20 I I.Alcaldía de Sibaté. Disponible en:<http:// www.sibate-cundinamarca.gov.co/apc-intranet>

Aranguren, G. (2000). Participación y ciudadanía. Madrid: PPC.

Boisier, S. (2002). Desarrollo territorial y descentralización: el desarrollo en el lugar y en las manos de la gente. Ministerio de la Presidencia del Perú.

Boisier, S. (2003). ¿Y si el desarrollo fuese una emergencia sistémica? Revista Reforma y Democracia, $N^{\circ} 27$, Caracas, CLAD. 
Britos, N. (1999) Ciudadanía y participación local. Universidad Nacional de Córdoba, Argentina.

Brugué, Q., Font, J., y Gomà, R. (sin año). Participación y democracia: asociaciones y poder local. Perú: Escuela de Gerencia Continental.

Budge, I. (1996). The New Challenge of Direct Democracy. Londres: Polity Press. Bogotá:Traducción, noviembre 2009.

Corragio, J. (2004). Perspectivas del desarrollo regional en América Latina. La gente o el capital. Argentina: Espacio.

Font, J. y Gomà, R. (2000). Participación Ciudadana en la Política Local. En Fundación Encuentro (ed.), Informe España 2000. Madrid: Fundación Encuentro.

Forero, C., Cardona, G., Córdoba, C. (1999). Planeación participativa, estrategia de paz. Bogotá: Consejo Nacional de Planeación

Garay, J. (2002). Democracia deliberativa y globalización: un esquema teórico de referencia. En (Ed.) Ciudadanía, lo público, democracia. Bogotá.

Gómez, Edisson (2009). Participación juvenil y administración pública: una aproximación a su forma ontológica. Revista Política \& administración, N 10 . Bogotá.

Grup d'Aprofundiment Democràtic, Mesa Cívica pels Drets Socials (2000). Participación ciudadana: reflexiones y propuestas. Catalunya.

Gyford, J. (199I). Citizens, Consumers and Councils. Gobierno local y lo público. Londres.

Held, D. (1992). Modelos de democracia. México:Alianza.

Hernández, H. (2008). Participación, democracia y gobernabilidad. En Administración y Desarrollo, No 49, ESAP.

Hopenhayn M. (1988). La Participación y sus Motivos. Acción Crítica. Lima: CELATS.

Hopenhayn, M. (2004). Participación Juvenil y política pública: un modelo para armar. Brasil:ALAP.

Kanbur, R., \& Squire, L. (1999). Evolución del pensamiento acerca de la pobreza: la búsqueda de las interacciones.

IIED - LA (2006) Inidencia e impacto social. Argentina: CENOC.

Kay, C. (2007). Enfoques sobre el desarrollo rural en América Latina y Europa desde mediados del siglo XX. En (Ed.) La enseñanza del desarrollo rural. Enfoques y perspectivas. Colombia: Universidad Javeriana.

Kendall, F. (1997). Democracia directa: la clave para un gobierno responsable. En Molteni y Krause (coord.), Democracia Directa. Argentina, Buenos Aires: Edit. Abeledo Perrot.

Kliksberg, B. (1999). Seis tesis no convencionales sobre la participación. Centro de documentación en políticas sociales.Argentina: Gobierno de la ciudad de Buenos Aires.

Max-Neef, M. (1997). Desarrollo sin sentido. En (ed.) Diseñadores del futuro. Colombia: Fundación Colombia multicolor.

Rebollo, O. (2003). Bases político-metodológicas para la participación. Boletín ciudades para un futuro más sostenible, No 24. España.

Rahnema,W. (1996). Participación. Ed., Diccionario del desarrollo. Una guía del conocimiento como poder. Perú: PRATEC.

Sen, A. (2000). El derecho a no tener hambre. Colombia: Universidad externado de Colombia.Traducción de Lamprea, E. 
Sckopol, T. (1995). El estado regresa al primer plano: estrategias de análisis en la investigación actual. Recuperado en noviembre de 2009, en biblioteca virtual de ciencias sociales, www.cholonautas.edu.pe

Subirats, J. (200I). Experiències de Participació Ciutadana en els Municipis Catalans. Barcelona: Escola d’Administració Pública de Catalunya. 